\section{ETIOLOGY AND DIFFERENTIAL DIAGNOSIS OF ADMITTED CHILDREN WITH HYPERTENSION}

\author{
Z.-D. Du, C. Liu \\ Beijing Children's Hospital, Capital Medical \\ University, Beijing, China
}

Objective: This study sought to summarize the clinical data of children who were admitted in Beijing Children's hospital with a diagnosis of hypertension, and try to analyze the discriminating factors between primary hypertension and secondary hypertension.

Methods: All children who were admitted to Beijing Children's Hospital during 2003 through 2007 were enrolled. Data on medical history, physical examination and laboratory studies were recorded. Hypertension was diagnosed according to the Chinese guideline for childhood hypertension and American national high blood pressure education program criteira. Hypertension was classified as primary and secondary based on the etiology of hypertension.

Results: A total of 304 children were included. Out of them, $146(48.0 \%)$ children were classified as primary hypertension, $158(52.0 \%)$ were secondary hypertension. Renal hypertension is the first etiology in secondary hypertension, followed by hypertension due to endocrine system diseases, cardiovascular system and central nervous system. Multiple logistic regression showed positive family history of hypertension (OR=8.897), age of older than 10 years $(O R=10.176)$ and increased $B M I$ $(\mathrm{OR}=19.256)$ were independent risk factors of primary hypertension, while ST-T changes on electrocardiogram $(\mathrm{OR}=0.0257)$, erecting serum renin angiotensin $>6 \mathrm{ng} / \mathrm{ml}$.h $(\mathrm{OR}=0.0593)$ were risk factors of secondary hypertension.

Conclusions: Secondary hypertension is more common in children admitted. Family history of hypertension, age of older than 10 years, increased BMI were risk factors of primary hypertension, while ST-T changes on electrocardiogram, erecting serum renin angiotensin $>6 \mathrm{ng} / \mathrm{ml}$.h were risk factors of secondary hypertension.

\section{THE IMPACT OF APROTININ IN ACUTE KIDNEY INJURY AFTER CARDIOPULMONARY BYPASS IN PEDIATRIC PATIENTS}

M.A. Gil-Ruiz Gil-Esparza1', A.J. Alcaraz Romero, J. del Castillo Peral ${ }^{1}$, J. Lorente Romero², A. Romero Otero ${ }^{1}$, A. Sánchez Galindo ${ }^{1}$, M.L. Franco Fernández ${ }^{2}$, E. Teigell Guerrero-Estracha ${ }^{3}$

${ }^{1}$ Servicio de Cuidados Intensivos Pediátricos, ${ }^{2}$ Servicio de Cuidados Intensivos Neonatales, ${ }^{3}$ Servicio de Anestesia Infantil, Hospital General Universitario Gregorio Marañon, Madrid, Spain

Background and aims: The use of aprotinin as antifibrinolytic agent in cardiopulmonary bypass (CPB) has been associated with increased postoperative morbidity and kidney damage in adults. Our goal was to evaluate the relationship between the antifibrinolytic used and development of postoperative renal damage in children.

Methods: Retrospective study including children undergoing CPBsurgeryduring 3years. Demographic variables, clinical and surgical-related aggressions and antifibrinolytic treatment used were collected. Kidney damage was assesed with pediatric RIFLE criteria (RIFLE-p). Data analysed with chi-square test and multivariate logistic regression.

Results: 403 patients were included. Median age: 7 months (18.1\% neonates). Clamping was used in 302 children and circulatory arrest (CA) in 131. The RACHS-1 score was $\geq 4$ in 101 patients. Aprotinin was used in 243, and tranexamic acid in 122 children.128 children showed kidney damage. 57 required renal replacement therapy. The group receiving aprotinin showed less kidney damage (27.3\% vs $39.5 \%, p=0.012)$. This group had shorter CPB (130 vs $151 \mathrm{~min}$; $p=0.006)$, less use of CA ( $23 \%$ vs $47 \%$ ), lower RACHS-1 (23\% vs $33 \%$ RACHS-1> 4) and included fewer infants (10\% vs $31 \%), p<0.001$. Controlling the effect of age and surgical aggression, no association between use of aprotinin and kidney damage was observed [OR 1.32, IC95\% (0.78-2.24), $p=0.291]$.

Conclusions: The antifibrinolytic treatment with aprotinin in extracorporeal circulation has not been associated with increased renal damage in our pediatric group. Further investigations are needed to evaluate the safety profile of aprotinin in children and its influence in renal failure. 